Pacific Journal of Mathematic 


\title{
ON THE PRIME IDEALS OF THE RING OF ENTIRE FUNCTIONS
}

\author{
MELVIN HENRIKSEN
}

1. Introduction. Let $R$ be the ring of entire functions, and let $K$ be the complex field. In an earlier paper [6], the author investigated the ideal structure of $R$, particular attention being paid to the maximal ideals. In 1946, Schilling [ 9, Lemma 5] stated that every prime ideal of $R$ is maximal. Recently, I. Kaplansky pointed out to the author (in conversation) that this statement is false, and constructed a nonmaximal prime ideal of $R$ ( see Theorem 1 (a), below). The purpose of the present paper is to investigate these nonmaximal prime ideals and their residue class fields. The author is indebted to Prof. Kaplansky for making this investigation possible.

The nonmaximal prime ideals are characterized within the class of prime ideals, and it is shown that each prime ideal is contained in a unique maximal ideal. The intersection $P^{*}$ of all powers of a maximal free ideal $M$ is the largest nonmaximal prime ideal contained in $M$. The set $P_{M}$ of all prime ideals contained in $M$ is linearly ordered under set inclusion, and distinct elements $P$ of $P_{M}$ correspond in a natural way to distinct rates of growth of the multiplicities of the zeros of functions $f$ in $P$.

It is shown that the residue class ring $R / P$ of a nonmaximal prime ideal $P$ of $R$ is a valuation ring whose unique maximal ideal is principal; $R / P$ is Noetherian if and only if $P=P^{*}$. The residue class ring $R / P^{*}$ is isomorphic to the ring $K\{z\}$ of all formal power series over $K$. The structure theory of Cohen [2] of complete local rings is used.

2. Notation and preliminaries. A familiarity with the contents of [6] is assumed, but some of it will be reproduced below for the sake of completeness.

DEFINITION 1. If $f \in R$, and $I$ is any nonvoid subset of $R$, let:

(a) $A(f)=[z \in K \mid f(z)=0]$ (Note that multiple zeros are repeated. Unions and intersections are taken in the same sense.);

(b) $A(I)=[A(f) \mid f \in I]$;

Received December 5, 1952.

Pacific J. Math. 3 (1953), 711-720 
(c) $A^{*}(f)$ be the sequence of distinct zeros of $f$, arranged in order of increasing modulus.

In 1940, Helmer showed [5, Theorem 9] that if $A(f) \cap A(g)$ is empty, there exist $s, t$ in $R$ such that

$$
s f+t g=1 .
$$

More generally, if $d$ is any element of $R$ such that

$$
A(d)=A(f) \cap A(g),
$$

then $d$ is a greatest common divisor of $f$ and $g$, unique to within a unit factor, and the ideal $(f, g)$ generated by $f$ and $g$ is the principal ideal $(d)$. It easily follows that every finitely generated ideal of $R$ is principal.

He proved this by showing that if $\left\{a_{n}\right\}$ is any sequence of complex numbers such that

$$
\lim _{n \rightarrow \infty} a_{n}=\infty
$$

and $w_{n, k}$ is any set of complex numbers, then there is an $s$ in $R$ such that

$$
s^{(k)}\left(a_{n}\right)=w_{n, k}, \quad\left(n=1,2, \cdots ; k=0, \cdots, 1_{n}\right) .
$$

The latter was shown independently by Germay [3].

REMARK. In [4], Germay extended (2.2) to the ring of functions analytic in $|z|<r$, where $\lim _{n \rightarrow \infty} a_{n}$ lies on $|z|=r$. Hence (2.1) follows for this ring, as will most of the results in [6] and the present paper, with minor modification.

It follows that if $I$ is an ideal of $R$, then $A(I)$ has the finite intersection property. So we make the following definition.

Definition 2. If $\bigcap_{f \in I} A(f)$ is nonempty, then $I$ is called a fixed ideal. Otherwise, $l$ is called a free ideal.

DEFINITION 3. (a) If $A^{*}(f)=\left\{a_{n}\right\}$, let $0_{n}(f)$ be the multiplicity of $a_{n}$ as a zero of $f$.

(b) If $A$ is a nonvoid subset of $A^{*}(f)$, let $0_{n}(f: A)$ be the function $0_{n}(f)$ with domain restricted to $A$.

(c) Let $m(f)=\sup _{n \geq 1} 0_{n}(f)$, if $f \neq 0$. Let $m(0)=\infty$.

3. Prime ideals of $R$. Kaplansky's construction of nonmaximal, prime ideals 
of $R$ is given in Theorem 1 (a), below. The only fallacy in Schilling's demonstration (referred to in the Introduction) is the false assumption that a prime ideal necessarily contains an $f$ such that $m(f)=1$. Hence a characterization of these nonmaximal prime ideals may be given.

THEOREM 1. (a) There exist nonmaximal prime ideals of $R$.

(b) A necessary and sufficient condition that a prime ideal $P$ of $R$ be nonmaximal is that $m(f)=\infty$, for all $f \in P$.

Proof. (a) Let

$$
S=[f \in R \mid m(f)<\propto]
$$

Clearly, $S$ is closed under multiplication and does not contain 0 . If $g \neq 0$ is in $R-S, g$ is contained in a prime ideal $P$ not intersecting $S$ (see [8, p.105]). Since, as noted in $[6$, p. 183], any maximal ideal contains an $f$ such that $m(f)=$ $1, P$ cannot be maximal.

(b) The sufficiency is clear from the above. If $f \in P$ with $m(f)<\propto$, the primality of $P$ ensures that there is a $g \in P$ with $m(g)=1$. Suppose the maximal ideal $M$ contains $P$, and let $h \in M$. By (2.1), there is a $d \in M$ such that

$$
A(d)=A(g) \cap A(h)
$$

Now $g=g_{1} d$, where $A\left(g_{1}\right) \cap A(d)$ is empty, since $m(g)=1$. Since $P$ is prime, it follows that either $g_{1} \in P$ or $d \in P$. But $M \neq R$, so $g_{1}$ is not in $P$. It follows that $d$, and hence $h$, is in $P$, whence $P=M$.

Corollary. Any prime, fixed ideal of $R$ is maximal.

THEOREM 2. Every prime ideal $P$ of $R$ is contained in a unique maximal ( free) ideal $M$.

Proof. By Theorem $1(\mathrm{~b})$ and [6, Theorem 4], the ideal $(P, f)$ is maximal if $m(f)=1$ and $A(f)$ intersects every element of $A(P)$. Let $f_{1}, f_{2}$ be any two such functions, so that $M_{1}=\left(P, f_{1}\right)$ and $M_{2}=\left(P, f_{2}\right)$ are maximal ideals containing $P$. If

$$
A(d)=A\left(f_{1}\right) \cap A\left(f_{2}\right),
$$

then $M=(P, d)$ is a maximal ideal containing $P$, and $M_{1} \subset M, M_{2} \subset M$, so that

$$
M_{1}=M_{2}=M
$$


More concrete constructions of nonmaximal prime ideals are given below in terms of maximal free ideals.

THEOREM 3. If $M$ is a maximal free ideal of $R$, then

$$
P^{*}=\bigcap_{k=1}^{\infty} M^{k}
$$

is a prime ideal, and is the largest nonmaximal prime ideal contained in $M$.

Proof. Since every finitely generated ideal of $R$ is principal, $P^{*}$ is easily seen to be the set of all $f \in R$ expressible in the form $h_{k} d_{k}^{k}$, with $d_{k} \in M, k=1$, $2, \cdots$. Thus, if $f \in M, f \in P^{*}$ if and only if $m(f / e)=\infty$ whenever $e$ divides $f$ and $e \in R-M$, (whence $f / e \in M$ ). Suppose $f_{1}, f_{2}$ are not in $P^{*}$. Clearly, $f_{1} f_{2}$ is not in $P^{*}$ except possibly when both $f_{1}$ and $f_{2}$ are in $M$. In this case, there exist $e_{i}$ dividing $f_{i}$, with $e_{i} \in R-M$ such that $m\left(f_{i} / e_{i}\right)<\infty,(i=1,2)$. Since $M$ is prime, $e_{1} e_{2} \in R-M$ and $m\left(f_{1} f_{2} / e_{1} e_{2}\right) \leq m\left(f_{1} / e_{1}\right)+m\left(f_{2} / e_{2}\right)<\infty$. So $f_{1} f_{2}$ is not in $P^{*}$, whence $P^{*}$ is a prime ideal.

The second part of the Theorem is a direct consequence of Theorem 1 (b).

We proceed now to identify the remainder of the class $P_{M}$ of prime ideals contained in $M$. This is done by considering the rates of growth of the functions $0_{n}(f)$ on the filter $A(M)$. Results of Bourbaki [1] are used without further acknowledgement.

DEFinition 4. If $f, g \in M$, and there is an $e \in M$ such that

$$
A^{*}(e) \subset A^{*}(f) \cap A^{*}(g)
$$

with

$$
0_{n}\left(f: A^{*}(e)\right) \geq 0_{n}\left(g: A^{*}(e)\right)
$$

then $f \geq g(g \leq f)$.

It is easily seen that the relation " $\geq "$ is reflexive and transitive. Moreover:

LeMma l. If $f, g \in M$, either $f \geq g$ or $g \geq f$.

Proof. Let

$$
A(d)=A(f) \cap A(g),
$$

and let 


$$
\begin{aligned}
& A_{1}=\left[z \in A^{*}(d) \mid 0_{n}(f:\{z\}) \geq 0_{n}(g:\{z\})\right], \\
& A_{2}=\left[z \in A^{*}(d) \mid 0_{n}(f:\{z\})<0_{n}(g:\{z\})\right] .
\end{aligned}
$$

Since $A_{1} \cap A_{2}$ is empty, $A_{1} \cup A_{2}=A^{*}(d)$; and since $M$ is prime, one and only one of $A_{1}, A_{2} \in M$. Hence $f \geq g$ or $g \geq f$.

DEF INITION 5. Suppose $f, g \in M$.

(a) If there exist positive integers $N_{1}, N_{2}$ such that $f^{N_{1}} \geq g$ and $g^{N_{2}} \geq f$, then $f \sim g$.

(b) If $f \geq g^{N}$ for all positive integers $N$ or if $f=0$, then $f \gg g(g<<f)$.

LEмма 2. (a) The relation ' $\sim$ ' is an equivalence relation.

(b) The relation ' $\gg$ ' is transitive.

(c) If $f, g \in M$, one and only one of $f \sim g, f \gg g, f \ll g$ holds.

Proof. The relations (a) and (b) follow easily from the observations that

$$
0_{n}\left(f^{N}\right)=N \cdot 0_{n}(f) \text {, and if } f \geq g \text { then } f^{N} \geq g^{N} .
$$

It is clear that at most one of the relations (c) can hold. By Lemma $1, f \geq g$ or $g \geq f$. Suppose $f \geq g$ and not $f \sim g$; then $f \geq g^{N}$ for all $N$, whence $f>g$. Similarly, if $g \geq f$.

Lemma 3. Let $f$ be an element of a prime ideal $P$ of $P_{M}$. If $g \geq f$, or $g \sim f$, then $g \in P$.

Proof. Suppose first that $g \geq f$. Then, as is evident from the construction in Lemma 1, we can write

$$
f=f_{1} d_{1}, g=g_{1} d_{2},
$$

where

$$
A^{*}\left(d_{1}\right)=A^{*}\left(d_{2}\right), \quad 0_{n}\left(d_{2}\right) \geq 0_{n}\left(d_{1}\right),
$$

and $f_{1}, g_{1}$ are not in $M$. Hence $d_{1} \in P$; and, since $d_{2}$ is a multiple of $d_{1}, d_{2}$ and $g \in P$. If $g \sim f$, then $g^{N} \geq f$, for some $N$. By the above, $g^{N} \in P$. But $P$ is a prime ideal, so $g \in P$.

THEOREM 4. (a) Let $\Omega$ be any subset of $M$, and let

$$
P_{\Omega}=[f \in M \mid f \gg g, \text { for all } g \in \Omega] \text {. }
$$


Then $P_{\Omega}$ is a prime ideal.

(b) If $P$ is a prime ideal, then $P=P_{\Omega}$, where $\Omega=M-P$.

Proof. (a) Note first that if $g_{1}, g_{2} \in M$ and $g_{1} g_{2} \neq 0$

$$
A=A^{*}\left(g_{1}\right) \cap A^{*}\left(g_{2}\right) \text {, }
$$

then

$$
0_{n}\left(g_{1}-g_{2}: A\right)=\min \left\{0_{n}\left(g_{1}: A\right), 0_{n}\left(g_{2}: A\right)\right\} .
$$

If $g_{1} \in M, g_{2} \in R, g_{1} g_{2} \neq 0$, then

$$
0_{n}\left(g_{1} g_{2}: A^{*}\left(g_{1}\right)\right)=0_{n}\left(g_{1}: A^{*}\left(g_{1}\right)\right)+0_{n}\left(g_{2}: A^{*}\left(g_{1}\right)\right) \text {. }
$$

It now follows from the lemmas above that $P$ is an ideal. The primality of $P$ follows from the observation that

$$
P_{g}=[f \in M \mid f>>g]
$$

is a prime ideal, and that $P_{\Omega}$ is an intersection of a descending chain (under set inclusion ) of ideals of this form.

(b) If $P$ is a prime ideal, the relations $f \in P, g \in M-P$, imply that $f \gg g$, by Lemma 3 .

COROLLARY. The ideals of $P_{M}$ are linearly ordered under set inclusion.

By the Theorem above, every element of $P_{M}$ is the upper class of a Dedekind cut ( under $\ll$ ). If $P$ contains a least element $f$, then

$$
P=P_{f}^{+}=[g \in M \mid g \gg f \text { or } g \sim f] .
$$

If $M-P$ has a greatest element $g$, then $P=P_{g}$ as defined in the proof of the theorem. It is clear that $P_{M}$ contains the greatest lower bound and least upper bound of any set of elements.

Note, moreover that $P_{f_{1}}=P_{f_{2}}\left(P_{f_{1}}^{+}=P_{f_{2}}^{+}\right)$if and only if $f_{1} \sim f_{2}$.

Lemma 4. The set $P^{*}-\{0\}$ has no countable cofinal or coinitial subset. Moreover, if $\left\{f_{1, n}\right\},\left\{f_{2, n}\right\}$ are two sequences of nonzero elements of $P^{*}$, such that

$$
f_{1, n+1} \gg f_{1, n} \gg f_{2, m} \gg f_{2, m+1}, \quad \text { for all } n, m,
$$

then there is an $f \in P^{*}$ such that 


$$
f_{1, n} \gg f \gg f_{2, m}, \quad \text { for all } n, m \text {. }
$$

Proof. See [1, p. 123, exercise 8].

The author is indebted to Dr. P. Erdös and Dr. L. Gillman for the following Theorem.

THEOREM 5. The set $P_{M}$ has power at least $2^{\aleph_{1}}$.

Proof. It is implicit in arguments of Hausdorff and Sierpinski $[10$, p.62] that every set satisfying Lemma 4 contains a subset similar to the lexicographically ordered set $S$ of $\omega_{1}$-sequences of 0 's and 1's, each having at most countably many l's By [11], $S$ is dense in the set of all dyadic $\omega_{1}$-sequences, which has power $2^{\aleph_{1}}$. Since the set $P_{M}$ is complete, card $\left(P_{M}\right) \geq 2^{\aleph_{1}}$.

Since card $\left(P_{M}\right) \leq 2^{c}$, where $c$ is the cardinal number of the continuum, we have:

CoROLlaRy. If $2^{\aleph_{1}}=2^{c}$, in particular if $\aleph_{1}=c$, then card $\left(P_{M}\right)=2^{c}$.

4. Residue class rings of prime ideals. We adopt the following definition of Krull [7, p. 110]:

DEF IN ITION 6. An integral domain $D$ such that if $f, g \in D$, then $f$ divides $g$ or $g$ divides $f$, is called a valuation ring.

It is easily seen that a valuation ring possesses a unique maximal ideal, consisting of all its nonunits.

THEOREM 6. The residue class ring $R / P$ of a prime ideal $P$ of $R$ is a valuaring whose unique maximal ideal is principal.

First, we prove a lemma.

Lemma 5. If $P \in P_{M}$, then $f$ is singular modulo $P$ if and only if $f \in M$.

Proof. Consider the equation

$$
f X \equiv 1(\bmod P)
$$

If $f \in M$, the equation clearly has no solution since $A(f) \cap A(p)$ is nonempty for all $p \in P($ see $[6$, Theorem 4$])$.

On the other hand, if $f$ is not in $M$, there is a $p \in P$ such that $A(f) \cap A(p)$ is empty. Let $A^{*}(p)=\left\{a_{n}\right\}$, with $0_{n}(p)=l_{n}$, in which case $f\left(a_{n}\right) \neq 0$. The 
equation in question has a solution if and only if there exists a $g \in R$ such that

(i) $g\left(a_{n}\right)=\left\{f\left(a_{n}\right)\right\}^{-1}$,

and

(ii) $(f g)^{(k)}\left(a_{n}\right)=0, k=1, \cdots, l_{n}$.

Since

$$
(f g)^{(k)}=f g^{(k)}+\sum_{i=1}^{k}\left(\begin{array}{c}
k \\
i
\end{array}\right) f^{(i)} g^{(k-i)}, \quad \text { where }\left(\begin{array}{c}
k \\
i
\end{array}\right)=\frac{k !}{i !(k-i) !},
$$

(ii) is satisfied if

(iii) $g^{(k)}\left(a_{n}\right)=-\left\{f\left(a_{n}\right)\right\}^{-1} \sum_{i=1}^{k}\left(\begin{array}{l}k \\ i\end{array}\right) f^{(i)}\left(a_{n}\right) g^{(k-i)}\left(a_{n}\right)$.

Such a $g$ can be constructed by (2.2), whence

$$
f g \equiv 1(\bmod P) .
$$

Proof of Theorem 6. By Lemma 5, every element of $R-M$ is a unit, so we may assume that $f, g \in M$. Let

$$
A(d)=A(f) \cap A(g),
$$

so that $A(f / d) \cap A(g / d)$ is empty. Clearly, at least one of $f / d, g / d \in R-M$, and hence is a unit modulo $P$. So $R / P$ is a valuation ring.

If, in particular, $f$ is chosen to be in $M-M^{2}, f / d$ cannot be in $M$, so $g$ is a multiple (modulo $P$ ) of $f$. Therefore the unique maximal ideal $M / P$ of $R / P$ is generated by $f$, and hence is principal.

If $P \neq P^{*}, R / P$ possesses the nonmaximal prime ideals $P_{1} / P$, where $P_{1}$ is a nonmaximal prime ideal of $R$ properly containing $P$. Moreover:

THE OREM 7. The residue class ring $R / P$ of a nonmaximal prime ideal $P$ is Noetherian if and only if $P=P^{*}$.

Proof. Every nonzero element of $M-P^{*}$ is in $M^{k}-M^{k-1}$, for some unique positive integer $k$. Hence every nonzero ideal of $R / P^{*}$ is of the form $\left(f^{k}\right)$, where $f \in M-M^{2}$.

If $f \in P-P^{*}$, construct $f_{k}$ such that 


$$
A^{*}\left(f_{k}\right)=A^{*}(f)
$$

and

$$
0_{n}\left(f_{k}\right)=\max \left\{0_{n}(f)-k, 1\right\}
$$

Then $f_{k+1}$ is a proper divisor (modulo $P$ ) of $f_{k}$. Hence the ideal generated by all the $f_{k}$ does not have finite basis.

The residue class ring $R / P^{*}$ is concretely identified below by the use of the structure theory of complete local rings [2] of Cohen. First we make a definition.

DEFINITION 7. (a) If the nonunits of a Noetherian ring $D$ with unit form a maximal ideal $M$ such that

$$
\prod_{k=1}^{\infty} M^{k}=(0),
$$

$D$ is called a local ring.

(b) If $f_{1}, \cdots, f_{n}$ is a minimal basis for $M$ such that $f_{1}, \cdots, f_{i}$ generate a prime ideal $(i=1, \ldots, n), S$ is called a regular local ring.

(c) Using the powers of $M$ as a system of neighborhoods of 0 , (thereby topologizing $D$ ), we call $D$ complete if every Cauchy sequence in $D$ has a (unique) limit.

THЕ ОR ЕМ 8. The residue class ring $R / P^{*}$ is isomorphic with the ring $K\{z\}$ of all formal power series over $K$.

Proof. By Theorems 3, 4, 6, $R / P^{*}$ is a local ring and is trivially regular since $M / P^{*}$ is principal. Cohen [2, Theorem 15] has shown that every regular, complete, local ring, whose unique maximal ideal is principal, and such that $D / M$ is isomorphic to $K$, is isomorphic to $K\{z\}$. By [6, Theorem 6],

$$
\left(R / P^{*}\right) /\left(M / P^{*}\right) \cong R / M \cong K
$$

The proof is completed by the following Lemma.

LЕммА 6. The residue class ring $R / P^{*}$ is complete.

Proof. Let $\left\{f_{k}\right\}$ be any Cauchy sequence in $R / P^{*}$. We may assume without loss of generality that $f_{k+1}-f_{k} \in M^{k}$, since a Cauchy sequence has at most one limit. Let 


$$
A_{k}=\left\{a_{k}, a_{k+1}, \cdots\right\} \in A(M)
$$

with all $a_{k}$ distinct. Let

$$
B_{k}=A_{k} \cap A\left(f_{k+1}-f_{k}\right)
$$

Clearly, $B_{k} \in A(M)$, and $\cap_{k=1}^{\infty} B_{k}$ is empty. Hence, we may construct by (2.2) an $f \in R$ such that

$$
f(z)=f_{1}(z) \quad \text { for } z \in B_{1} \text {, }
$$

and

$$
f^{(k)}(z)=f_{k}^{(k)}(z) \quad \text { for } z \in B_{k+1} \text {. }
$$

Then

$$
f_{k} \equiv f\left(\bmod M^{k}\right)
$$

whence

$$
L_{k \rightarrow \infty} f_{k}=f
$$

\section{REFERENCES}

1. N. Bourbaki, Étude locale des fonctions, Actualités Sci. Ind., No. 1132, Hermann et Cie., Paris, 1951.

2. I. S. Cohen, On the structure and ideal theory of complete local rings, Trans. Amer. Math. Soc. 59 (1946), 54-106.

3. R. H. J. Germay, Sur une application des théorèmes de Weierstrass et de Mittag* Leffler de la théorie générale des fonctions, Ann. Soc. Sci. Bruxelles, Ser. I, 60 (1946), 190-195.

4. - Extension d'un théorème de E. Picard relatif aux produits indéfinis de facteurs primaires, Bull. Roy. Sci. Liège 17 (1948), 138-143.

5. O. Helmer, Divisibility properties of integral functions, Duke Math. J. 6 (1940), 345-356.

6. M. Henriksen, On the ideal structure of the ring of entire functions, Pacific J. Math. 2 (1952), 179-184.

7. W. Krull, Idealtheorie, Ergebnisse der Mathematik, Julius Springer, Berlin, 1935.

8. N. McCoy, Rings and ideals, Mathematical Association of America, Buffalo, 1950.

9. O. F. G. Schilling, Ideal theory on open Riemann surfaces, Bull. Amer. Math. Soc. 52 (1946), 945-963. 56-67.

10. W. Sierpiński, Sur une propriété des ensembles ordonnés, Fund. Math. 36 (1949),

11. - Sur un problème concernant les sous-ensembles croissants du continu, Fund. Math. 3 (1922), 109-112.

Purdue University 


\title{
PACIFIC JOURNAL OF MATHEMATICS
}

\author{
EDITORS
}

\author{
R. M. BOEINSON \\ University of California \\ Berkeley 4, California \\ E. HEWITT \\ University of Washington \\ Seattle 5, Washington
}

\author{
P. P. DILWOR TH \\ California Institute of Technology \\ Pasadena 4, California \\ E. F. BECKENBACH \\ University of California \\ Los Angeles 24, California
}

\section{ASSOCIATE EDITORSS}

$\begin{array}{llll}\text { H. BUSEMANN } & \text { P. R. HALMOS } & \text { BØRGE JESSEN } & \text { J. J. STOKER } \\ \text { HERBERT FFDERER } & \text { HEINZ HOPF } & \text { PAUL LÉVY } & \text { E. G. STR AUS } \\ \text { MARSHALL HALI } & \text { R. D. JAMES } & \text { GEORGE PÓLYA } & \text { KÔSAKU YOSIDA }\end{array}$

\section{SFONSORS}

UNIVERSITY OF BRITISH COLUMBIA

CALIFORNIA INSTITUTE OF TECHNOLOGY.

UNIVERSITY OF CAIIFORNIA, BERKELEY

UNIVERSITY OF CALIFORNIA, DAVIS

UNIVERSITY OF CALIFORNIA, LOS ANGELES

UNIVERSITY OF CALIFORNIA, SANTA BARBARA

UNIVERSITY OF NEVADA

OREGON STATE COLLEGE

UNIVERSITY OF OREGON

\author{
UNIVERSITY OF SOU THERN CALIFORNIA \\ STANFORD RESEARCH INSTITUTE \\ STANFORD UNIVERSITY. \\ WASHINGTON STATE COLLEGE \\ UNIVERSITY OF WASHINGTON \\ AMERICAN MATHEMATICAL SOCIETY \\ NATIONAL BUREAU OF STANDARDS, \\ INSTITUTE FOR NUMERICAL ANALYSIS
}

Mathematical papers intended for publication in the Pacific Journal of Mathematics should be typewritten (double spaced), and the author should keep a complete copy. Manuscripts may be sent to any of the editors-except Robinson, whose term expires with the completion of the present volume; they might also be sent to M.M. Schiffer, Stanford University, Stanford, California, who is succeeding Robinson. All other communications to the editors should be addressed to the managing editor, $\mathrm{E}$. F. Beckenbach, at the address given above.

Authors are entitled to receive 100 free reprints of their published papers and may obtain additional copies at cost.

The Pacific Journal of Mathematics is published quarterly, in March, June, September, and December. The price per volume (4 numbers) is $\$ 8.00$; single issues, $\$ 2.50$. Special price to individual faculty members of supporting institutions and to individual members of the American Mathematical Society: $\$ 4.00$ per volume; single issues, $\$ 1.25$.

Subscriptions, orders for back numbers, and changes of address should be sent to the publishers, University of California Press, Berkeley 4, California.

Printed at Ann Arbor, Michigan. Entered as second class matter at the Post Office, Berkeley, California.

\author{
UNIVERSITY OF CALIFORNIA PRESS - BERKELEY AND LOS ANGELES
}




\section{Pacific Journal of Mathematics}

\section{Vol. 3, No. $4 \quad$ June, 1953}

Paul Erdős and Gilbert Agnew Hunt, Jr., Changes of sign of sums of random variables........................................ 673

Paul Erdôs and Ernst Gabor Straus, On linear independence of sequences in

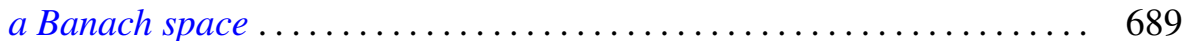

Haim Hanani, On sums of series of complex numbers ............... 695

Melvin Henriksen, On the prime ideals of the ring of entire functions ..... 711

Irving Kaplansky, Completely continuous normal operators with property L............................................. 721

Samuel Karlin, Some random walks arising in learning models. I. ...... 725

William Judson LeVeque, On uniform distribution modulo a subdivision ..................................... 757

Lee Lorch, Derivatives of infinte order ..................... 773

Ernest A. Michael, Some extension theorems for continuous functions . . . . . 789

Tyre Alexander Newton, A note on the Hölder mean ................ 807

Raymond Moos Redheffer, On a theorem of Plancherel and Pólya....... 823

Choy-Tak Taam, On the complex zeros of functions of Sturm-Liouville

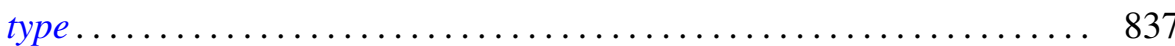

